\title{
The inheritance of anthocyanin pigmentation in potato (Solanum tuberosum L.) and mapping of tuber skin colour loci using RFLPs
}

\author{
HERMAN J. VAN ECK*, JEANNE M. E. JACOBS †, PETRA M. M. M. VAN DEN BERG, \\ WILLEM J. STIEKEMA † AND EVERT JACOBSEN \\ Department of Plant Breeding, Wageningen Agricultural University, P.O. Box 386, NL-6700 AJ Wageningen and \\ $\uparrow$ Department of Molecular Biology, DLO-Centre for Plant Breeding and Reproduction Research (CPRO-DLO), P.O. BOX \\ 16, NL-6700 AA Wageningen, The Netherlands
}

\begin{abstract}
Two existing genetic models for anthocyanin pigmentation are compared: the genetic model as proposed by Lunden $(1937,1960,1974)$ for tetraploid potato Solanum tuberosum group Tuberosum and the model by Dodds \& Long $(1955,1956)$ for diploid cultivated Solanum species. By crossing well defined genotypes from both genetic sources it was demonstrated that locus $R / R^{p w}$ and locus $D$ are allelic. Both loci are involved in the biosynthesis of red anthocyanins. We propose to maintain the symbol $D$ for this locus. Tuber skin colour is expressed due to the complementary action of one locus involved in anthocyanin biosynthesis and a second locus involved in tissuespecific regulation of anthocyanin expression. The inheritance of two phenotypes of skin colour was investigated in this study: pigmentation of the epidermis and pigmentation of the cortex. In two different populations the loci determining the two phenotypes were located with RFLPs on the map of the potato genome, at approximately the same position as the earlier mapped PSC locus on chromosome 10. Lunden proposed the symbols $E$ and $R$ for the loci determining these phenotypes. Dodds and Long proposed only one locus $I$ for tuber skin colour without describing these different phenotypes. The identity and nomenclature of the loci studied by the various authors are discussed.
\end{abstract}

Keywords: allelism, morphological markers, RFLPs, Solanum tuberosum, tuber skin colour.

\section{Introduction}

Genetic research on the potato was started at the beginning of this century by Salaman (1910) with a study on the inheritance of tuber skin colour. Salaman. postulated three unlinked loci to explain the phenotypes of purple, red and white tubers. Red pigmentation of tubers is the result of the complementary action of the loci $D$ and $R$ which both show dominance. Together with factor $P$ they give tubers a purple skin colour. Later, locus $E$ was added to this model to explain another phenotype of skin colour pigmentation (Salaman, 1926; Lunden, 1937). Locus $E$ is involved in the pigmentation of the epidermis and the eyes of the tuber, whereas locus $R$ is expressed in the cortex. The studies on the inheritance of anthocyanin pigmentation in tetraploid European varieties in the first half of this century were reviewed by Von Rathlef \& Siebeneick

${ }^{*}$ Correspondence.
(1934) and by Swaminathan \& Howard (1953) and is presently known as the genetic model for group Tuberosum $(2 n=4 \mathrm{x}=48)$.

Without much reference to previous work, Dodds \& Long $(1955,1956)$ developed another genetic model for the inheritance of anthocyanin pigmentation in diploid cultivated species $(2 n=2 \mathrm{x}=24)$. The loci $P$, $R / R^{p w}, B, I$ and $F$ were postulated to explain anthocyanin pigmentation in various parts of the plant. Reviews by Howard (1970) and De Jong (1991) tried to compare the loci postulated for group Tuberosum and loci postulated for diploid cultivated species. Experimental evidence on assumed allelic relations between the two genetic models has not been presented until now.

Efficient methods for the production of dihaploid clones from tetraploid varieties (Hermsen \& Verdenius, 1973) allow genetic studies of group Tuberosum germplasm at the diploid level without the complication of tetrasomic inheritance. At the diploid 
level, clones representing group Tuberosum germplasm can be hybridized with diploid cultivated species. The development of RFLP technology and the publication of detailed genetic maps of the potato genome (Bonierbale et al., 1988; Gebhardt et al., 1989, 1991) have greatly stimulated and facilitated genetic studies in potato.

At present, several loci involved in the inheritance of classical morphological traits are mapped. Bonierbale et al. (1988) mapped locus $Y$, involved in flesh colour, on chromosome 3, and Gebhardt et al. (1989) mapped a purple skin colour locus (PSC) on chromosome 10 . In our previous publications we mapped the flower colour locus $F$ on chromosome 10 and the loci $D$ and $P$, involved in anthocyanin biosynthesis, on chromosomes 2 and 11, respectively (Van Eck et al., 1993), and the Ro locus for tuber shape on chromosome 10 (Van Eck et al., 1994).

This study had two objectives:

1 demonstrating allelism for loci belonging to two different genetic models for anthocyanin pigmentation, which were postulated for group Tuberosum and diploid cultivated species, respectively, and

2 investigating the inheritance of two different phenotypes for tuber skin colour and mapping the loci involved on the genetic map of the potato.

\section{Materials and methods}

\section{Nomenclature in the genetic model for group Tuberosum and description of the phenotypes}

The genetic model for group Tuberosum germplasm was proposed by Salaman (1910) and extended by Lunden (1937). The postulated loci $D, P$ and $F$ have been located on the genetic map of potato (Van Eck et al., 1993).

Locus D; chromosome 2. A locus with dominance involved in the biosynthesis of red pigments throughout the plant. Locus $D$ is complementary to the flower and skin colour loci $F, E$ and $R$. Without the presence of dominant alleles at these flower and skin colour loci, it is still possible to detect the presence of a dominant $D$ allele by the weak brownish-red pigmentation of the sprout tips, stems, flower stalks and calyx (Lunden, 1937).

Locus $\mathrm{P}$; chromosome 11. A locus with dominance involved in the biosynthesis of blue pigments throughout the plant, especially visible in hypocotyl and sprout tips. Like locus $D$, the $P$-locus is complementary to the loci $F, E$ and $R$ in the expression of flower and tuber skin colour (Lunden, 1937).
Locus E. A locus with dominance regulating the presence of red or purple pigmentation in the epidermis of the tuber in combination with locus $D$ or $P$, respectively. Plants with genotype $p p d d E$. still have a weak, diluted reddish colour in the tubers, with stronger pigmentation in the eyes and at the bases of the sprouts.

Locus R. A locus with dominance regulating the presence of pigmentation in the outer layers of the cortex of the tuber; no colour in the epidermis. In combination with either locus $D$ or $P$, red or blue-purple tubers are produced. Genotype P.D.R. gives an intensely pigmented tuber cortex with a black appearance. The almost white tuber skin phenotype of $p p d d R$. is difficult to distinguish from white-skinned D.rr or P.rr genotypes (Lunden, 1937). Pigmentation of the tuber can be located by stripping off the epidermis of freshly harvested tubers.

Locus $\mathrm{F}$; chromosome 10. A locus with dominance regulating the presence of flower colour. Genotype $p p D . F$. gives red-purple flowers, $P . d d F$. pale-blue flowers, P.D.F. blue-purple flowers and $p p d d F$. or ....ff gives white flowers (Lunden, 1937).

\section{Nomenclature of the genetic model for diploid cultivated species and description of phenotypes}

Dodds \& Long $(1955,1956)$ developed the genetic model for diploid cultivated species.

Locus $\mathrm{R} / \mathrm{R}^{\mathrm{pw}} \quad$ In the absence of $P$, this locus controls the production of red pigments in tubers, flowers and sprouts. The homozygous recessive genotype $R^{p w} R^{p w}$ has pink tubers and sprouts but white flowers. A recessive allele $r$ giving complete absence of pigmentation has never been described in diploid cultivated species (Dodds \& Long, 1955).

Locus P. This locus controls the formation of blue pigments throughout a diploid potato plant. In tubers, locus $P$ is epistatic to locus $R / R^{p w}$; in flowers, epistasis is incomplete (Dodds \& Long, 1955).

Locus I. The genotype $i i$ at locus $I$ causes absence of pigmentation of the tubers (Dodds \& Long, 1956). Genotypes $P . R^{p w} R^{p w} i i$ and $p p R . i i$ give, according to this model, purple and red sprouts respectively with white tuber skin.

Locus F. A locus with a flower-specific expression involved in the contrast of self-coloured vs. flecked, homozygous recessives $f f$ being flecked. Genotypes which are recessive at locus $R / R^{p w}$ show white flowers (Dodds \& Long, 1956). 


\section{Pedigree and genotypes of plant materials}

Group Tuberosum germplasm is represented by three diploid tester genotypes $\mathrm{I}_{1} \mathrm{G} 254-26, \mathrm{I}_{1} \mathrm{G} 254-77$ and $\mathrm{I}_{1} \mathrm{G} 254-83$ derived from the dihaploid G254 from the group Tuberosum cultivar cv. Gineke (Van Eck et al., 1993). The genotypes of these tester clones with white flowers and tubers are indicated in Table 1, following the nomenclature by Lunden. Alleles in this material are identical by descent to those involved in previous studies by Lunden (1974). The genotypes of parental clones 7553-1, 79-48 and 7506-1 are described (De Jong, 1987) according to the nomenclature of Dodds \& Long $(1955,1956)$. The clones are derived from an Agriculture Canada Breeding Project, Fredericton, NB, involving the diploid species $S$. stenotomum and $S$. phureja. Cross 79-48 $\times 7506-1$ gave progeny $88-405$ which segregated for red pigmentation of the epidermis of the tuber; cross 7553-1 $\times 7506-1$ gave progeny 88 402 which segregated for blue-purple pigmentation of the cortex of the tuber. The genotypes of the clones, representing loci for anthocyanin pigmentation from diploid cultivated species, are also shown in Table 1 . Individual genotypes of the progenies $88-405$ and 88-
402 were crossed as female parents with the earlier mentioned tester clones of group Tuberosum used as male parents.

\section{Procedures to detect DNA polymorphisms}

Isolation of genomic DNA from young leaves and shoots was as described by Bernatzky \& Tanksley (1986). Restriction digestions were performed with the enzymes EcoRI, HindIII and $X b a \mathrm{I}$ using 2 units per $\mu \mathrm{g}$ DNA, according to the manufacturers' instructions (Amersham). Fragments, 6-8 $\mu \mathrm{g}$ DNA per lane, were separated in a 0.8 per cent TBE-buffered agarose gel and transferred to Hybond $\mathrm{N}$ membrane by capillary blotting using a $1 \mathrm{M} \mathrm{NH}_{4} \mathrm{OH} / 20 \mathrm{~mm} \mathrm{NaOH}$ transfer solution. Blots were hybridized against digoxigenindUTP labelled DNA probes. Hybridization mixtures with labelled probe can be reused several times for a period of at least 1 year. Signal detection was performed with anti-dig-AP antibodies (Boehringer) and AMPPD (Tropix) as substrate for chemiluminescence according to Kreike et al. (1990). Luminograms were obtained within $2 \mathrm{~h}$ by placing the membranes on $\mathrm{X}$-ray film (Kodak X-Omat). After stripping the probe

Table 1 Description of the skin colour phenotypes and genotypes of the parental potato (Solanum tuberosum L.) clones

\begin{tabular}{|c|c|c|c|}
\hline \multirow[b]{2}{*}{ Potato clones } & \multirow[b]{2}{*}{ Phenotypes } & \multicolumn{2}{|c|}{ Genotypes nomenclature } \\
\hline & & Group Tuberosum & $\begin{array}{l}\text { Diploid cultivated } \\
\text { species }\end{array}$ \\
\hline \multicolumn{4}{|l|}{ Parental clones } \\
\hline cv. Gineke* & $\operatorname{Red}(\mathbf{L} 2)$ & ppppDDddeeeeRrrrFff & \\
\hline $\mathrm{G} 254 \dagger$ & White & ppDdeerrFf & \\
\hline $\mathrm{I}_{1} \mathrm{G} 254-26 \ddagger$ & White & ppddeerrFF & \\
\hline $\mathrm{I}_{1} \mathrm{G} 254-77 \ddagger$ & White & ppDDeerrff & \\
\hline$I_{1} G 254-83 \ddagger$ & White & ppddeerrff & \\
\hline $79-48 \S$ & $\operatorname{Red}(\mathbf{L} 1)$ & & $p p R R^{p w} I I$ \\
\hline $7553-1 \S$ & Purple (L2) & & $P p R R^{p w} I i$ \\
\hline $7506-1 \S$ & White & & $p p R^{p w} R^{p w} i i$ \\
\hline \multicolumn{4}{|c|}{ Genotypes selected from the cross $79-48 \times 7506-1 \pi$} \\
\hline $88-405-1$ & $\operatorname{Red}(\mathbf{L} 1)$ & & $p p R R^{p w} I i$ \\
\hline $88-405-3$ & Pink & & $p p R^{p w} R^{p w} I i$ \\
\hline $88-405-5$ & $\operatorname{Red}(\mathbf{L} 1)$ & & $p p R R^{p w} I i$ \\
\hline $88-405-8$ & Pink & & $p p R^{p w} R^{p w} I i$ \\
\hline $88-405-12$ & Pink & & $p p R^{p w} R^{p w} I i$ \\
\hline $88-405-38$ & Pink & & $p p R^{p w} R^{p w} I i$ \\
\hline \multicolumn{4}{|c|}{ Genotypes selected from the cross $7553-1 \times 7506-1 \pi$} \\
\hline $88-402-12$ & Purple (L2) & & $P p R^{p w} R^{p w} I i$ \\
\hline $88-402-25$ & White & & $p p R R^{p w i i}$ \\
\hline
\end{tabular}

*Lunden, 1974; †Hermsen et al., 1978; †Van Eck et al., 1993; §De Jong, 1987; Tthis article.

$\mathrm{L} 1$ and $\mathrm{L} 2$ refer to epidermis and cortex, respectively.

The genotype is given in the nomenclature belonging to the origin of the germplasm. 
from the blot, reuse of the blots is possible at least five times. When too much background signal limits further use of the blots with the chemiluminescence procedure, it is still possible to use the blot in a radioactive procedure.

The map positions of the RFLP marker loci have previously been published by Gebhardt et al. (1989, 1991: GP and CP clones), Bonierbale et al. (1988: TG clones) and Kreike et al. (1993: Ssp clone).

\section{Segregation, linkage analysis and map construction}

Only the female parents 88-405-5 and 88-402-12 segregated at RFLP loci, and the male 'inbred' parent $I_{1}$ G254-77 appeared to be homozygous at all loci tested. Consequently, the genetic maps are based on recombination events in the female meiosis. The computer program JOINMAP (Stam, 1993) calculated the most likely map configuration, using the mapping function of Kosambi (1944). The computer program DRAWMAP (Van Ooijen, 1994) was used to draw the maps from this study and previous data (Van Eck et al., $1993,1994)$ referring to the flower colour locus $F$ (Lunden, 1937) and the tuber shape locus Ro (Masson, 1985 ) as shown in Fig. 1. Raw RFLP data are loaded in the nightshade database which is available via anonymous ftp from: probe.nalusda.gov in the directory pub/ solgenes, or via plant genome gopher SolGenes. (For

Fig. 1 Genetic maps of potato (Solanum tuberosum L.) chromosome 10 (Kosambi distances in $\mathrm{cM}$ ). (a) Genetic map of clone 88-405-5 showing the tuber skin colour locus $I_{e p}$ involved in pigmentation of the epidermis. (b) Genetic map of clone 88-40212 showing the tuber skin colour locus $I_{c o}$ involved in pigmentation of the cortex. (c) Genetic map of potato showing flower colour locus $F$ (Van Eck $e t$ al., 1993) and tuber shape locus $R o$ (Van Eck et al., 1994). (d) Joined map of potato chromosome 10 showing the positions of morphological marker loci. more information: internet: Epaul@nightshade.cit. cornell.edu).

\section{Results}

\section{Phenotypes of tuber skin colour in the segregating} progenies 88-405 and 88-402

Progenies $88-405$ of the cross $79-48 \times 7506-1$ and 88 402 of the cross $7553-1 \times 7506-1$ represent genetic material of diploid cultivated species (De Jong, 1987). The progeny 88-405 segregated into descendants with red and pink tubers in a 19:13 ratio (this fits an expected ratio of $1: 1 ; \chi_{1}^{2}=1.13$ ) due to heterozygosity -at the $R / R^{p w}$ locus in the female parent 79-48. The clones of 88-405 with red tubers and red flowers have the genotype $p p R R^{p w} I i$, and descendants with pink tubers and white flowers have the genotype $p p R^{p w} R^{p w} I i$ (Table 1). These observations are in agreement with the genetic model for diploid cultivated species (Dodds \& Long, 1955) and with the parental genotypes postulated by De Jong (1987): the pistillate parent 79-48 with red flowers and red tubers has the genotype $p p R R^{p w} I I$; the unpigmented staminate parent 7506-1 has the genotype $p p R^{p w} R^{p w} i i$.

The segregation observed in progeny 88-402, derived from $7553-1 \times 7506-1$, is also in agreement with the parental genotypes postulated by De Jong

(b) $88-402-12$

(c) $\mathrm{C} \times \mathrm{E}$

(d) JOINED MAP

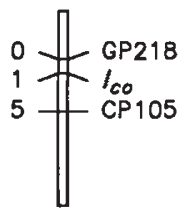

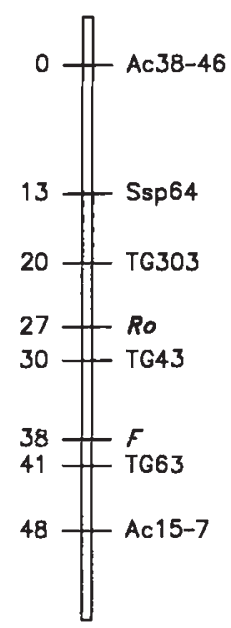

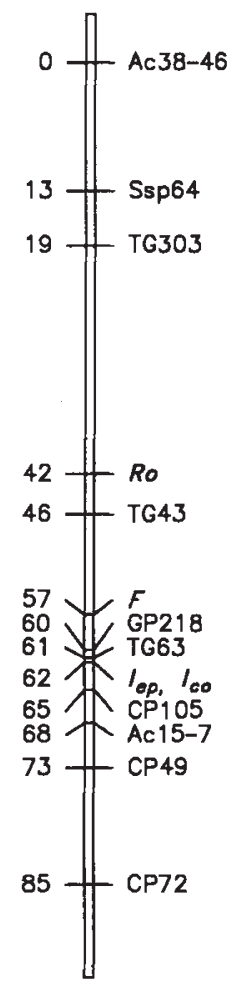


(1987) and the genetic model for diploid cultivated species. Heterozygosity of the pistillate parent 7553-1 at loci $P, R / R^{p w}$ and $I$ is confirmed by the observation of purple, red and white-skinned descendants, and purple, red and white pigmentation of the sprouts within the descendants with white tuber skin colour.

The red skin colour of 88-405 descendants was observed in the epidermis of the tuber. In contrast to this phenotype the red or purple skin colour segregating in 88-402 clones is located in the outer cell layers of the cortex. The genetic model for anthocyanin pigmentation in diploid cultivated species (Dodds \& Long, 1955) does not discriminate between these two phenotypes, whereas the genetic model for tetraploid group Tuberosum has two different loci, $E$ and $R$, to explain these phenotypes.

The phenotype of the offspring clones 88-405-.. with pink tubers and white flowers having genotype $p p R^{p w} R^{p w} I i$ matches well with the phenotype of $d d E e$ as described by Lunden (1937). This phenotype is described as having a weak, diluted, reddish colour in the tubers, with stronger pigmentation in the eyes and at the base of the sprouts (Lunden, 1937). The resemblance suggests that skin colour locus $I$ (Dodds \& Long, 1955) in this material, derived from diploid cultivated species, may be allelic with the skin colour locus $E$ (Lunden, 1937) from tetraploid group Tuberosum germplasm. The segregation of pigmentation in the tuber epidermis as well as in the flower due to locus $R / R^{p w}$ resembles the action of locus $D$ (Lunden, 1937) which is involved in the production of red pigments throughout the plant (Van Eck et al., 1993). Therefore, allelism between locus $R / R^{p w}$ (Dodds \& Long, 1955) and locus $D$ (Lunden, 1937) is tested.

In 88-402 clones, where pigmentation was located in the cortex of the tubers, the effect of locus $I$ (Dodds \& Long, 1955) is similar to the effect of a second skin colour locus $R$ as described by Lunden (1937). Among the descendants of 88-402 clones, the typical pink tubers with red eyes as observed in the previous cross due to skin colour locus $E$ were not observed, indicating another difference between these skin colour loci. $p p R^{p w} R^{p w} I i$ descendants from 88-402-12 had almost white tubers and white flowers, but could be distinguished from true white $p p R^{p w} R^{p w} i i$ genotypes on the basis of pigmentation of the sprout tips. These phenotypical observations suggest allelism between the skin colour locus $I$ (Dodds \& Long, 1955) and the skin colour locus $R$ (Lunden, 1937) from tetraploid group Tuberosum material.

\section{The inheritance of flower and tuber skin colour in 88- 405 genotypes}

To examine allelic relations between the loci postulated in diploid cultivated species and group Tuberosum, the 88-405-clones were hybridized with the tester clones $I_{1} G 254-26, I_{1} G 254-77$ and $I_{1} G 254-83$ with white flowers and tubers. Clone $\mathrm{I}_{1} \mathrm{G} 254-77$ with genotype ppDDeerrff will transmit to its progeny the dominant $D$ allele which is involved in the biosynthesis of red anthocyanins. Tester clone $\mathrm{I}_{1} \mathrm{G} 254-26$ with genotype ppddeerrFF will transmit a dominant $F$ allele which is involved in the flower-specific expression of pigmentation. The complementary action of the loci $D$ and $F$ will result in the presence of red flower colour. Clone $\mathrm{I}_{1} \mathrm{G} 254-83$ is recessive at loci $P, D$ and $F$ (Van Eck et al., 1993), and at the loci $E$ and $R$. Observations on flower and tuber skin colour of hybrid progenies between 88-405 and the tester clones are presented in the Tables 2 and 3, respectively.

No difference in segregation was observed for either flower colour or tuber skin colour between descendants of clone $I_{1}$ G254-26 and $I_{1}$ G254-83. However, when tester $\mathrm{I}_{1} \mathrm{G} 254-77$ was used, which contributes the $D$ allele to the progeny, pink tuber skin and white flowers were found. Recessiveness at locus $R / R^{\mathrm{pw}}$, resulting in pink tubers and white flowers, can be supplemented in diploid cultivated species with a dominant allele of locus $D$ originating from group Tuberosum. This clearly demonstrates allelism between locus $R / R^{p w}$ and locus $D$.

From Table 2 the effect of a dominant $F$ allele can be inferred from hybrids between 88-405 clones and tester clone $\mathrm{I}_{1} \mathrm{G} 254-26$ in comparison with hybrids between $88-405$ and $\mathrm{I}_{1} \mathrm{G} 254-83$. Because there is no difference in the presence or segregation ratio of flower colour in descendants of both $I_{1}$ G254-83 and $I_{1}$ G25426 it is concluded that $88-405$ individuals are uniformly homozygous dominant at the $F$ locus.

Although locus $I$ segregates in progenies from all 88-405 clones, the data in Table 2 demonstrate that flower colour, as expected, is not affected by the segregating locus $I$ involved in tuber skin colour. The presence or absence of flower colour and the expected ratios for this trait can be adequately explained from the genotype at locus $R / R^{p w}$ in the female $88-405$ parent.

Four phenotypes of tuber pigmentation could be observed in the progeny of the crosses 88-405$1 \times \mathrm{I}_{1} \mathrm{G} 254-26,88-405-5 \times \mathrm{I}_{1} \mathrm{G}_{2} 54-26$ and 88-405$5 \times \mathrm{I}_{1} \mathrm{G} 254-83$ (Table 3). The tuber skin colour classes red, pink and white, are easily recognized. Subsequently, within the class with white tubers the segregation at locus $D$ can be recognized by the presence of either the flower colour or the pigmentation of the inflorescence or the sprout. However, sprout pigmentation is also affected by locus $E$, but in a different way. Sprouts of Ddee genotypes are pigmented mainly at the tip (characteristic No.7; UPOV, 1986). ddEe genotypes have pigmentation of the sprout mainly at the base (characteristic No.4; UPOV, 1986). In DdEe 
Table 2 Segregation of flower colour in crosses between germplasm of diploid cultivated species ( $88-405$ clones) and group Tuberosum $\left(\mathrm{I}_{1} \mathrm{G} 254\right.$-tester clones)

\begin{tabular}{|c|c|c|c|c|c|c|c|c|}
\hline \multicolumn{2}{|c|}{ Parents } & \multicolumn{2}{|c|}{ Phenotype } & \multicolumn{2}{|c|}{ Observed } & \multirow{2}{*}{$\begin{array}{l}\text { Expected* } \\
\text { Red:White }\end{array}$} & \multicolumn{2}{|c|}{ Parental genotypes } \\
\hline Female & Male & Female & Male & $\begin{array}{l}\text { Red } \\
D d . .\end{array}$ & $\begin{array}{c}\text { White§ } \\
d d . .\end{array}$ & & Female $\nmid$ & Male $\ddagger$ \\
\hline $88-405-1$ & $\mathrm{I}_{1} \mathrm{G} 254-26$ & Red & White & 13 & 16 & $1: 1$ & $R R^{p w} I i$ & ddeeFF \\
\hline $88-405-1$ & $\mathrm{I}_{1} \mathrm{G} 254-77$ & Red & White & 14 & 0 & $1: 0$ & $R R^{p w} I i$ & DDeeff \\
\hline $88-405-3$ & $I_{1} G 254-26$ & White & White & 0 & 23 & $0: 1$ & $R^{p w} R^{p w} I i$ & ddeeFF \\
\hline $88-405-3$ & $\mathrm{I}_{1} \mathrm{G} 254-77$ & White & White & 12 & 0 & $1: 0$ & $R^{p w} R^{p w} I i$ & DDeeff \\
\hline $88-405-3$ & $\mathrm{I}_{1} \mathrm{G} 254-83$ & White & White & 0 & 24 & $0: 1$ & $R^{p w} R^{p w} I i$ & ddeeff \\
\hline $88-405-5$ & $\mathrm{I}_{1} \mathrm{G} 254-26$ & Red & White & 16 & 14 & 1:1 & $R R^{p w} I i$ & ddeeFF \\
\hline $88-405-5$ & $\mathrm{I}_{1} \mathrm{G} 254-77$ & Red & White & 15 & 0 & 1:0 & $R R^{p w} I i$ & DDeeff \\
\hline $88-405-5$ & $\mathrm{I}_{1}^{2} \mathrm{G} 254-83$ & Red & White & 11 & 7 & $1: 1$ & $R R^{p w} I i$ & ddeeff \\
\hline $88-405-8$ & $\mathrm{I}_{1} \mathrm{G} 254-26$ & White & White & 0 & 48 & $0: 1$ & $R^{p w} R^{p w} I i$ & ddeeFF \\
\hline $88-405-8$ & $\mathrm{I}_{1} \mathrm{G} 254-77$ & White & White & 22 & 0 & $1: 0$ & $R^{p w} R^{p w} I i$ & DDeeff \\
\hline $88-405-8$ & $\mathrm{I}_{1} \mathrm{G} 254-83$ & White & White & 0 & 27 & $0: 1$ & $R^{p w} R^{p w} I i$ & ddeeff \\
\hline $88-405-12$ & $I_{1} G 254-26$ & White & White & 0 & 6 & $0: 1$ & $R^{p w} R^{p w} I i$ & ddeeFF \\
\hline $88-405-12$ & $\mathrm{I}_{1} \mathrm{G} 254-77$ & White & White & 16 & 0 & $1: 0$ & $R^{p w} R^{p w} I i$ & DDeeff \\
\hline $88-405-12$ & $I_{1} G 254-83$ & White & White & 0 & 33 & $0: 1$ & $R^{p w} R^{p w} I i$ & ddeeff \\
\hline $88-405-38$ & $\mathrm{I}_{1} \mathrm{G} 254-26$ & White & White & 0 & 20 & $0: 1$ & $R^{p w} R^{p w} I i$ & ddeeFF \\
\hline $88-405-38$ & $I_{1} G 254-77$ & White & White & 12 & 0 & $1: 0$ & $R^{p w} R^{p w} I i$ & DDeeff \\
\hline $88-405-38$ & $\mathrm{I}_{\mathrm{I}} \mathrm{G} 254-83$ & White & White & 0 & 13 & $0: 1$ & $R^{p w} R^{p w} I i$ & ddeeff \\
\hline
\end{tabular}

*All observations are in agreement with the expected ratios.

†Genotype symbols according to the nomenclature common for diploid cultivated species (Dodds \& Long, 1955, 1956).

$\ddagger$ Genotype symbols according to the nomenclature common for group Tuberosum (Lunden, 1937).

§The genotypes of the descendants belonging to the classes: red, DdEeF./DdeeF.; white, ddEeF./ddeeF. in the nomenclature common for group Tuberosum; red, $R R^{p w} I i / R R^{p w} i i$; white, $R^{p w} R^{p w} I i / R^{p w} R^{p w} i i$ in the nomenclature common for cultivated diploid species.

genotypes, sprout colour is red both at the base and the tip. The genotype may also be deduced from the sprout phenotype for the other crosses mentioned in Table 3.

The pink tuber skin colour is only observed in $d d E e$ e genotypes. This indicates that the pink pigments are the effect of a dominant $E$ allele ( $=I$ allele) and not the result of a recessive $d$ allele ( $=R^{p w}$ allele). According to the description by Dodds and Long (1955), the effect of locus $I$ would be as a recessive inhibitor of pigments produced by the recessive $R^{p w}$ allele. Genetically, this may be a correct interpretation, but from a molecular point of view, it is paradoxical to ascribe a positive function to a recessive allele.

In Table 3 it is shown that tuber skin colour is not influenced by locus $F$. This is in agreement with our expectations as locus $F$ is involved in the expression of flower colour.

The following points should be noted regarding the observations in Tables 2 and 3. Firstly, for a specific cross the progeny size for flower colour is not equal to the progeny size for tuber skin colour. This is because not all seedlings flowered or set tubers. Secondly, the red flowering descendants segregated 1:1 for red and white tuber skin colour, and the white flowering ones segregated into pink and white tuber skin colour. Both segregations are due to the segregation of locus $I$.

\section{RFLP mapping of the locus involved in skin colour in the epidermis}

The progeny of 88-405-5 $\times \mathrm{I}_{1} \mathrm{G} 254-77$, comprising 64 descendants, was used to map skin colour. In this population cosegregation was observed between skin colour and tuber shape $(17.2 \pm 4.7$ per cent recombination; $\chi_{1}^{2}=27.8 ; \mathrm{LOD}=6.5$ ). The $R o$ locus involved in tuber shape is located on chromosome 10 (Van Eck et al., 1994). Additionally, it was known that the PSC locus involved in purple skin colour was mapped on potato chromosome 10 (Gebhardt et al., 1989). This 
Table 3 Segregation of tuber skin colour in crosses between germplasm of diploid cultivated species (88-405 clones) and group Tuberosum $\left(\mathrm{I}_{1} \mathrm{G} 254\right.$-tester clones $)$

\begin{tabular}{|c|c|c|c|c|c|c|c|c|c|c|}
\hline \multicolumn{2}{|c|}{ Parents } & \multicolumn{2}{|c|}{ Phenotype } & \multicolumn{4}{|c|}{ Observed } & \multirow{2}{*}{$\begin{array}{c}\text { Expected* } \\
\text { r:w:p:w }\end{array}$} & \multicolumn{2}{|c|}{ Parental genotypes } \\
\hline Female & Male & Female & Male & $\begin{array}{l}\text { Red } \\
\text { DdEe }\end{array}$ & $\begin{array}{l}\text { White } \\
\text { Ddee }\end{array}$ & $\begin{array}{l}\text { Pink } \\
d d E e\end{array}$ & $\begin{array}{c}\text { White } \$ \\
\text { ddee }\end{array}$ & & Female $\dagger$ & Male $\ddagger$ \\
\hline $88-405-1$ & $\mathrm{I}_{1} \mathrm{G} 254-26$ & Red & White & 21 & 20 & 13 & 32 & 1:1:1:1 & $R R^{p w} I i$ & ddeeFF \\
\hline $88-405-1$ & $\mathrm{I}_{1} \mathrm{G} 254-77$ & Red & White & 13 & 37 & 0 & 0 & $1: 1: 0: 0$ & $R R^{p w} I i$ & DDeeff \\
\hline $88-405-3$ & $\mathrm{I}_{1} \mathrm{G} 254-26$ & Pink & White & 0 & 0 & 45 & 36 & $0: 0: 1: 1$ & $R^{p w} R^{p w} I i$ & ddeeFF \\
\hline $88-405-3$ & $\mathrm{I}_{1} \mathrm{G} 254-77$ & Pink & White & 41 & 38 & 0 & 0 & $1: 1: 0: 0$ & $R^{p w} R^{p w} I i$ & DDeeff \\
\hline $88-405-3$ & $\mathrm{I}_{1} \mathrm{G} 254-83$ & Pink & White & 0 & 0 & 26 & 38 & $0: 0: 1: 1$ & $R^{p w} R^{p w} I i$ & ddeeff \\
\hline $88-405-5$ & $I_{1} G 254-26$ & Red & White & 17 & 15 & 28 & 14 & 1:1:1:1 & $R R^{p w} I i$ & ddeeFF \\
\hline $88-405-5$ & $\mathrm{I}_{1} \mathrm{G} 254-77$ & Red & White & 38 & 34 & 0 & 0 & $1: 1: 0: 0$ & $R R^{p w} I i$ & DDeeff \\
\hline $88-405-5$ & $\mathrm{I}_{1} \mathrm{G} 254-83$ & Red & White & 21 & 18 & 21 & 13 & 1:1:1:1 & $R R^{p w} I i$ & ddeeff \\
\hline $88-405-8$ & $I_{1} G 254-26$ & Pink & White & 0 & 0 & 35 & 45 & $0: 0: 1: 1$ & $R^{p w} R^{p w} I i$ & ddeeFF \\
\hline $88-405-8$ & $I_{1} G 254-77$ & Pink & White & 34 & 37 & 0 & 0 & $1: 1: 0: 0$ & $R^{p w} R^{p w} I i$ & DDeeff \\
\hline $88-405-8$ & $\mathrm{I}_{1} \mathrm{G} 254-83$ & Pink & White & 0 & 0 & 45 & 38 & $0: 0: 1: 1$ & $R^{p w} R^{p w} I i$ & ddeeff \\
\hline $88-405-12$ & $\mathrm{I}_{1} \mathrm{G} 254-26$ & Pink & White & 0 & 0 & 48 & 34 & $0: 0: 1: 1$ & $R^{p w} R^{p w} I i$ & ddeeFF \\
\hline $88-405-12$ & $\mathrm{I}_{1} \mathrm{G} 254-77$ & Pink & White & 45 & 49 & 0 & 0 & $1: 1: 0: 0$ & $R^{p w} R^{p w} I i$ & DDeeff \\
\hline $88-405-12$ & $\mathrm{I}_{1} \mathrm{G} 254-83$ & Pink & White & 0 & 0 & 26 & 24 & $0: 0: 1: 1$ & $R^{p w} R^{p w} I i$ & ddeeff \\
\hline $88-405-38$ & $\mathrm{I}_{1} \mathrm{G} 254-26$ & Pink & White & 0 & 0 & 48 & 44 & $0: 0: 1: 1$ & $R^{p w} R^{p w} I i$ & ddeeFF \\
\hline $88-405-38$ & $\mathrm{I}_{1} \mathrm{G} 254-77$ & Pink & White & 40 & 40 & 0 & 0 & 1:1:0:0 & $R^{p w} R^{p w} I i$ & DDeeff \\
\hline $88-405-38$ & $\mathrm{I}_{1} \mathrm{G} 254-83$ & Pink & White & 0 & 0 & 36 & 48 & $0: 0: 1: 1$ & $R^{p w} R^{p w} I i$ & ddeeff \\
\hline
\end{tabular}

*All observations are in agreement with the expected ratios.

†Genotype symbols according to the nomenclature common for diploid cultivated species (Dodds \& Long, 1955, 1956).

‡Genotype symbols according to the nomenclature common for group Tuberosum (Lunden, 1937).

$\S$ The genotypes assigned to descendants belonging to the classes: red, white, pink, white are in the nomenclature common for group Tuberosum. The alternative notation would have been: red, white, pink, white $=R R^{p w} I i, R R^{p w} i i, R^{p w} R^{p w} I i, R^{p w} R^{p w} i i$.

information gave a strong indication about the possible map position of this tuber skin colour locus. Using RFLP markers from chromosome 10 it was possible to locate skin colour. In 63 observations, only one recombination was found between the skin colour locus and RFLP marker GP218 at one side (1.59 per cent recombination; $\left.\chi_{1}^{2}=59.1 ; \mathrm{LOD}=16.7\right)$ and another recombination between the skin colour locus and CP105 at the other side ( 1.59 per cent recombination; $\chi_{1}^{2}=59.1$; $\mathrm{LOD}=16.7$ ). The resulting map of cross 88-405$5 \times \mathrm{I}_{1} \mathrm{G} 254-77$, including several other RFLP markers, is shown in Fig. 1(a). The tuber skin colour locus is indicated with symbol $I_{e p}$ because the allele is descended from diploid cultivated species and allelism with locus $E$ (Lunden, 1937) is not demonstrated. The suffix indicates that the effect of this allele is observed in the epidermis of the tuber.

\section{The inheritance of flower and tuber skin colour in 88- 402 genotypes}

In this material, skin colour is based on cortex pigmentation, not the epidermis, and therefore the tetra- ploid symbol $R / r$ has to be used. Due to poor flowering, female sterility or low berry set only three combinations with the tester clones could be obtained. The segregations observed in these three progenies are presented in Table 4. The results of cross 7553$1 \times 7506-1$ are not fully described because $16\left(2^{4}\right)$ genotypes resulting in 10 different phenotypes are expected. With respect to tuber skin colour alone, the observed segregation is in close agreement with the expected ratio.

The most striking observation in this material is the absolute correlation between flower and skin colour in the progenies of $88-402-12 \times \mathrm{I}_{1} \mathrm{G} 254-77,88-402-$ $12 \times \mathrm{I}_{1} \mathrm{G} 254-83$ and $88-402-25 \times \mathrm{I}_{1} \mathrm{G} 254-83$ (Table 4). The correlation between flower and skin colour was particularly curious, because clones with pigmented tubers always had white flowers and clones with pigmented flowers always had white tubers. Initially, Lunden (1937) described independent loci for tuber and skin colour. Much later (Lunden, 1960), he discovered close linkage between skin colour locus $R$ and flower colour locus $F$, although this conclusion was weakened in his latest publication(Lunden, 1974). The 


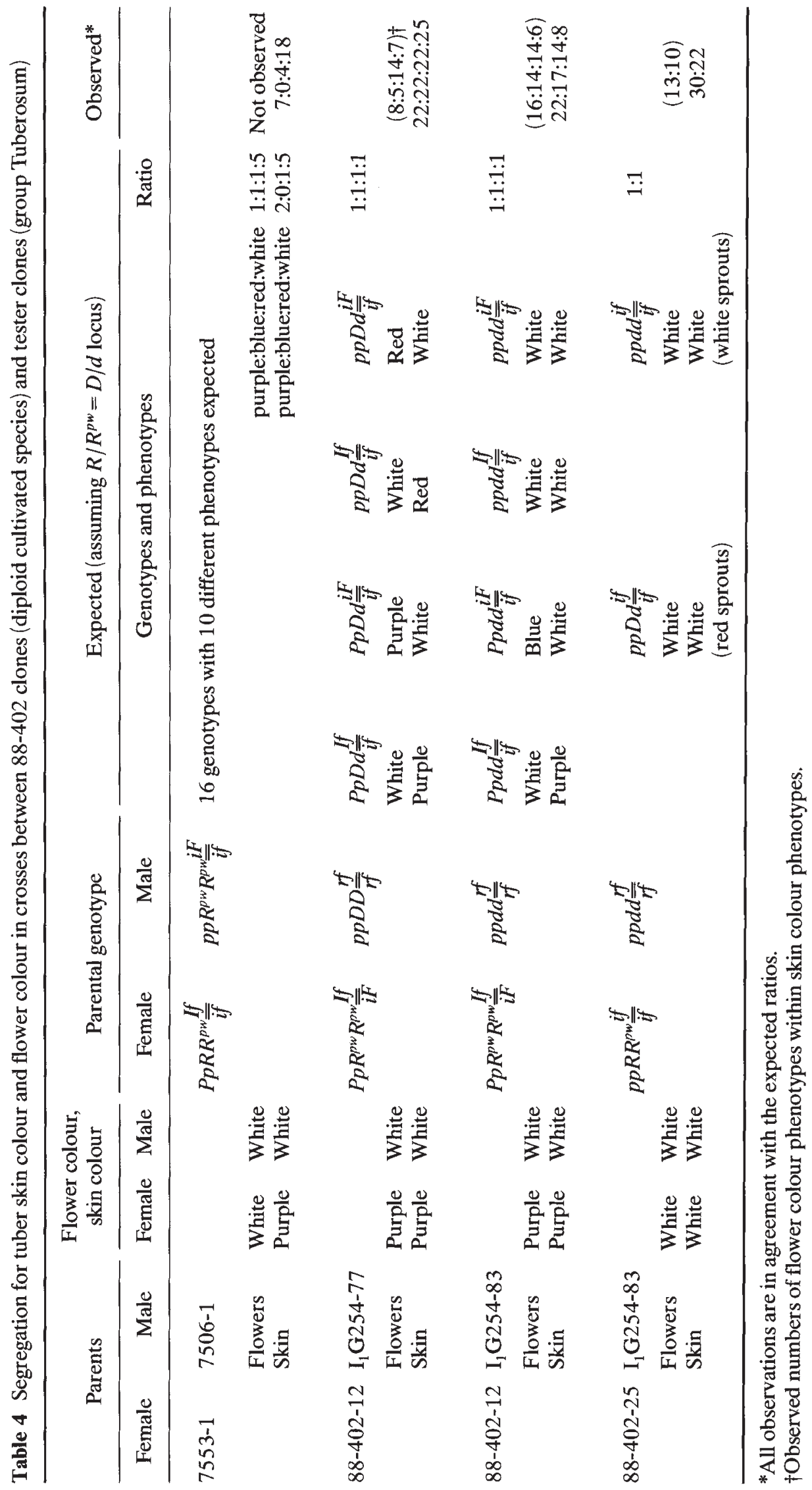


correlations reported in the literature always describe individuals where skin and flower colour are both present or absent. Dodds \& Long (1956) described a linkage group comprising three loci $B-I-F$. However, the $F$ locus as described by Dodds \& Long (1956) is assumed to be involved in the contrast of flecked vs. self-coloured flowers.

To explain the correlation found in descendants of 88-402-12, we propose linkage in the repulsion phase between the skin colour and flower colour loci. This explanation is a combination of the $B-I-F$ linkage group postulated for diploid cultivated species (Dodds \& Long, 1956) and the phenotype of locus $F$ as described by Lunden (1937) and its complementary action with locus $D$ (Lunden, 1937; Van Eck et al., 1993). Flower and tuber skin colour are independent in 7553-1 $\times 7506-1$ because the dominant alleles of locus $I$ and $F$ descend from the different parents.

When the progenies of 88-402-12 $\times \mathrm{I}_{1} \mathrm{G} 254-77$ and $88-402-12 \times I_{1} G 254-83$ are compared it is possible to characterize the effect of the dominant $D$ allele contributed by $\mathrm{I}_{1} \mathrm{G} 254-77$. Locus $D$ is involved in the ability to produce red anthocyanins throughout the plant, but tuber skin colour and flower colour are only observed in the presence of a complementary allele at locus $I$ or $F$. Without complementary gene action red pigments produced by locus $D$ can be observed in the tip of the sprouts, but not in the tuber skin or flowers.

Observations on skin and flower colour in the offspring of 88-402-12 demonstrate epistasic dominance of locus $P$ over locus $D$. This epistasis is complete in the tuber, but incomplete in the flower. This was also described for the interaction between loci $P$ and $R / R^{p w}$ (Dodds \& Long, 1955).

The conclusion of allelism between locus $R / R^{p w}$ and locus $D$ as drawn from the results shown in Tables 2 and 3 is also confirmed by the results in Table 4 .

The presence of a dominant allele at locus $D$ in clone 88-402-25 was inferred from the presence of red-pigmented flesh and red pigmentation of the tip of the spouts. Heterozygosity at locus $D$ was inferred from the 1:1 segregation for pigmented sprout tips in its offspring.

\section{RFLP mapping of the locus involved in skin colour in the cortex}

The offspring of 88-402-12 $\times \mathrm{I}_{1} \mathrm{G} 254-77$, comprising 91 descendants, were used to map the locus causing pigmentation of the cortex. Cosegregation was found between skin colour and RFLP marker CP105 with three recombinants out of 74 observations (4.1 per cent recombination; $\chi_{1}^{2}=62.5$; $\mathrm{LOD}=16.8$ ). Cosegregation was also observed with marker GP218 with only one recombinant out of 62 observations (1.6 per cent recombination; $\chi_{1}^{2}=58.1$; $\mathrm{LOD}=16.4$ ). These RFLP markers encompass the skin colour locus as the largest recombination fraction was found between CP105 and GP218 (3 out of 59, 5.1 per cent recombination; $\chi_{1}^{2}=47.7$; $L O D=12.6$ ). The locus involved in pigmentation of the cortex, probably locus $R$, appeared to be located on chromosome 10 at approximately the same position as the skin colour locus involved in pigmentation of the epidermis. Because allelism is not demonstrated between the locus segregating in this material derived from diploid cultivated species and locus $R$ involved in pigmentation of the cortex in tetraploid group Tuberosum (Lunden, 1937) the position of this locus is indicated with symbol $I_{c o}$ on the map shown in Fig. 1(b). The suffix indicates that this allele is involved in pigmentation of the cortex.

\section{Construction of an integrated map}

The two separate maps based on 88-405-5 and 88402-12 were integrated with data from previous publications (Van Eck et al., 1993, 1994) referring to the map position of flower colour locus $F$ (Lunden, 1937) and the tuber shape locus Ro (Masson, 1985) (Fig. 1c). The order of loci as described in Gebhardt (1991) was also taken into account by adding a fixed sequence file (GP218-TG63-CP105) to the computer program JoINMAP This integrated map of chromosome 10 (Fig. 1d) shows a short distance between the skin colour gene(s) at locus $I$ and the flower colour locus $F$ (Lunden, 1937). This provides further evidence that the linkage group $B-I-F$ (Dodds \& Long, 1956) is allelic to the linkage reported by Lunden (1960) between flower colour locus $F$ and skin colour locus $R$ in tetraploid group Tuberosum germplasm.

\section{Discussion}

\section{Evidence for allelism}

In this report the relationships were examined between two genetic models, proposed by different investigators for different germplasms. The loci and/or phenotypes were compared to find evidence for allelism between loci, which can be studied by three different methods. The first approach is based on the phenotype of the trait. The similarity between two phenotypes is a strong indication that these traits are controlled by the same locus, but this is no evidence for allelism. A second and much stronger procedure to test allelism is on the basis of the position of the locus on the genetic map. Loci with distinct map positions are not allelic. However, when two traits can be located on approximately the same 
locus, it is difficult to distinguish between allelism and close linkage of different loci. The third and reliable strategy to test for allelism is genetic complementation. This method requires crosses with well-defined tester genotypes.

Allelism between locus $D$ (Lunden, 1937) and locus $R / R^{\text {pw }}$ (Dodds \& Long, 1955) was demonstrated with the third method using the diploid tester clones derived from cv. Gineke. Also on the basis of phenotypic observations, the effects of loci $D$ and $R / R^{p w}$ are identical. Allelism between locus $D$ (Lunden, 1937) located on chromosome 2 (Van Eck, 1993) and I (Dodds \& Long, 1956) located on chromosome 10 (this report) as suggested by De Jong (1991), is rejected.

The tuber skin colour loci present in 88-405-5 and 88-402-12 and the PSC locus (Gebhardt et al., 1989) were mapped on chromosome 10 . The phenotype of the PSC locus is similar to the phenotype observed in clone 88-405-5 (C. Gebhardt, pers. comm.). The small difference between the map order reported in Gebhardt et al. $(1989,1991)$ and the one in this study can be explained as an artefact due to integration of loci which are polymorphic in only one parent. Integration of the female and male meioses is based on loci which are polymorphic in both parents. These loci serve as 'allelic bridges' between separate parental maps. Even when all loci are at the same chromosomal position, the different phenotypes caused by locus $E$ and locus $R$ can be due to different but closely linked genes.

The hypothesis of two closely linked skin colour genes is supported by many other closely linked loci involved in tissue-specific expression of anthocyanin pigmentation (Kessel \& Rowe, 1974; De Jong, 1987), including flower colour (Fig. 1) in this specific region of potato chromosome 10 .

\section{Evaluation of the genetic model as proposed by Lunden (1937, 1960, 1974)}

The extensive studies by Lunden have been a major achievement for potato genetics, particularly because the genetic studies were carried out with tetraploid varieties. Major drawbacks of the tetrasomic inheritance are the complex segregation ratios and the difficulties involved in establishing linkage relationships.

The weak aspect of the genetic model proposed by Lunden has been the contradictory information about correlation between flower and skin colours. To detect linkage between flower and skin colour loci it is necessary that the loci are simplex and that the dominant alleles are in linkage phase. When they are in repulsion phase or not in the simplex condition, a large progeny size is required to detect linkage (Wu et al., 1991). From the $\sim 100$ crosses investigated by Lunden (1937), only in six cases did the parent have a simplex genotype at the flower and skin colour loci. Additionally, the segregation of the anthocyanin-producing loci $D$ or $P$ interfered with the segregation of flower and skin colour. It is, therefore, not unlikely that genetic linkage between separate flower and skin colour loci has been overlooked.

Lunden (1937) proposed a model incorporating loci involved in the production of red or blue anthocyanins which are active throughout the plant, as well as loci controlling tissue-specific expression of these anthocyanins. This complementary inheritance of pigmentation is a very important aspect of the genetic model. The presence of complementary loci was confirmed by studies at the diploid level (Van Eck et al., 1993).

\section{Evaluation of the genetic model as proposed by Dodds \& Long (1955, 1956)}

The genetic model for diploid cultivated species has recognized the linkage between genetic loci for flower and skin colours. This linkage is confirmed by RFLP mapping of a flower colour locus (Van Eck et al., 1993) and a skin colour locus (Gebhardt et al., 1989; this study) on chromosome 10 . The different skin colour phenotypes were not described for diploid cultivated species. The evidence for maintaining two different skin colour genes at one genetic locus $I$ is based on differences in phenotype. Many different genetic loci involved in tissue-specific expression of anthocyanins are located at this small region on chromosome 10 (Dodds \& Long, 1956; De Jong \& Rowe, 1972; Kessel \& Rowe, 1974; De Jong, 1987). This clustering of loci involved in pigmentation is another argument in support of the presence of two different skin colour genes at locus $I$. The detection of genetic linkage between flower and skin colour loci is most likely due to the greater genetic resolution at the diploid level rather than to differences between the loci involved in anthocyanin pigmentation.

A major difference between the models seems to be the monogenic inheritance of colour traits in diploid cultivated species and the complementary inheritance of pigmentation as described by Lunden. However, from the description by Dodds \& Long (1956) of the inheritance of white, flecked and self-coloured flowers it can be deduced that in this material also flower colour is the result of complementary action of the $F$ and $R / R^{p w}$ loci. According to Dodds \& Long (1956) the $F$ locus is involved in the contrast between selfcoloured and flecked flowers. The tester genotype 
$\mathrm{I}_{1} \mathrm{G} 254-77$ with genotype $D D f f$ can produce pigments throughout the plant. Pigmentation can be observed in the sprouts, stem and calyx but also some pigmentation is expressed in the tips of the petals, in a pattern similar to that shown by Dodds \& Long (1956) for flecked flowers. Our interpretation is as follows: $R . F$. $=$ selfcoloured flowers; $R$.ff=flecked flowers, which are white flowers with some pigmentation due to leaky alleles of $f f, R^{p w} R^{p w} F$. and $R^{p w} R^{p w} f f=$ white flowers. Complementary inheritance is also present for tuber skin colour but again it is not described as such. The action of locus $I$ is described as an inhibitor in the homozygous recessive state (Dodds \& Long, 1956), rather than as a locus where the dominant allele is involved in the expression of skin colour. Locus $I$ is dependent on locus $R / R^{p w}$ for its full expression.

\section{Nomenclature}

To avoid further confusion between the nomenclature used in the different genetic models it is proposed to revoke the symbol $R / R^{p w}$ and to indicate the locus involved in the biosynthesis of red anthocyanins with the symbol $D$ : first, because locus $D$ was already proposed by Salaman (1910), long before the introduction of locus $R / R^{p w}$; and second, because the description of this locus as given by Dodds \& Long (1955) is not correct. Pink tuber colour is not the phenotypic expression of the recessive $R^{p w}$ allele, but that of the dominant $I$ allele in the absence of a dominant $D$ allele.

Four different names, $R, E, I$ and $P S C$ have been used for a locus on chromosome 10 involved in tuber skin colour. It is justified to revoke the symbol PSC (Gebhardt et al., 1989). This symbol was only used because it was unknown whether the segregation for skin colour was due to segregation of a locus involved in anthocyanin production or of a locus for tissuespecific expression. Because as yet no evidence is presented in favour of two different genetic loci, it is not possible to conclude whether the older names $R$ and $E$ or the more often used, but relatively new, symbol $I$ is to be preferred. Because genetic stocks used in this study were derived from diploid cultivated species, the symbol $I$ was used in Fig. 1 to indicate the skin colour locus. The differences between the skin colour alleles tracing back to the clones 88-405-5 and 88-402-12 are indicated by the suffixes $I_{e p}$ and $I_{c o}$.

\section{Acknowledgements}

The research was financially supported by the Netherlands Technology Foundation (STW), and was coordinated by the Foundation for Life Sciences (SLW). AnnekeThea Hertog, Jan Rijksen and Teus van den
Brink are gratefully acknowledged for their assistance in the greenhouse, and John Hermsen for critically reading the manuscript. Seeds from crosses 79$48 \times 7506-1$ and $7553-1 \times 7506-1$ were kindly provided by Dr H. De Jong. We thank Dr. C. M. Kreike, Dr C. Gebhardt and Dr S. D. Tanksley for kindly supplying the RFLP DNA clones.

\section{References}

BERNATZKY, R. AND TANKSLEY, S. D. 1986. Methods for detection of single or low copy sequences in tomato on Southern blots. Plant Mol. Biol. Rep., 4, 37-41.

BONIERBALE, M. W., PLAISTED, R. L. AND TANKSLEY, S. D. 1988. RFLP maps based on a common set of clones reveal modes of chromosomal evolution in potato and tomato. Genetics, 120, 1095-1103.

DE JONG, H. 1987. Inheritance of pigmented tuber flesh in cultivated diploid potatoes. Am. Potato J., 64, 337-343.

DE JONG, H. 1991. Inheritance of anthocyanin pigmentation in the cultivated potato: a critical review. Am. Potato J., 68, 585-593.

DE JONG, H. AND ROWE, P. R. 1972. Genetic markers in inbred clones of cultivated diploid potatoes. Potato Res., 15, 200-208.

DODDS, K. S. AND LONG, D. H. 1955. The inheritance of colour in diploid potatoes. I. Types of anthocyanidins and their genetic loci. J. Genet., 53, 136-149.

DODDS, K. S. AND LONG, D. H. 1956. The inheritance of colour in diploid potatoes. II. A three-factor linkage group. J. Genet., 54, 27-41.

GEBHARDT, C., RITTER, E., SCHACHTSCHABEL, U., WALKEMEIER, B., UHRIG, H. AND SALAMINI, F. 1989. RFLP analysis and linkage mapping in Solanum tuberosum. Theor. Appl. Genet., 78, 65-75.

GEBHARDT, C., RITTER, E., BARONE, A., DEBENER, T., WALKEMEIER, B., SCHACHTSCHABEL, U., KAUFMANN, H., THOMPSON, R. D., BONIERBALE, M. W., GANAL, M. W., TANKSLEY, S. D. AND SALAMINI, F. 1991. RFLP maps of potato and their alignment with the homoeologous tomato genome. Theor. Appl. Genet., 83, 49-57.

HERMSEN, J. G. Th. AND VERDENIUS, J. 1973. Selection from Solanum tuberosum Group Phureja of genotypes combining high frequency haploid induction with homozygosity for embryo-spot. Euphytica, 22, 244-259.

HERMSEN, J. G. Th., TAYLOR, L. M., VAN BREUKELEN, E. W. M. AND LIPSKI, A. 1978. Inheritance of genetic markers from two potato dihaploids and their respective parent cultivars. Euphytica, 27, 681-687.

Howard, H. W. 1970. Genetics of the Potato Solanum tuberosum. Logos Press, London.

KESSEL, R. AND ROWE, P. R. 1974. Inheritance of two qualitative traits and a proposed genetic map for their linkage group in diploid potatoes. Potato Res., 17, 283-295.

KoSAMBI, D. D. 1944. The estimation of map distances from recombination values. Ann. Eugen., 12, 172-175. 
KREIKE, C. M., DE KONING, J. R. A. AND KRENS, F. A. 1990. Non-radioactive detection of single-copy DNA-DNA hybrids. Plant Mol. Biol. Rep., 8, 172-179.

KREIKE, C. M., DE KONING, J. R. A., VINKE, J. H., OOIJEN, J. W. VAN, GEBHARDT, C. AND STIEKEMA, w. J. 1993. Mapping of loci involved in quantitatively inherited resistance to the potato cyst nematode Globodera rostochiensis pathotype Ro1. Theor. Appl. Genet., 87, 464-470.

LUNDEN, A. P. 1937. Arvelighetsundersøkelser i potet (Inheritance studies in the potato), Solanum tuberosum. Sœrtrykk av Meldinger fra Norges Landbrukshøiskole, Norges Landbrukshøiskoles Åkervekstforsøk.

LUNDEN, A.P. 1960. Some more evidence of autotetraploid inheritance in the potato (Solanum tuberosum). Euphytica, 9, 225-234.

LUNDEN, A. P. 1974. Inheritance of tuber and flower colour in the potato (Solanum tuberosum). Meldinger fra Norges landbrukshøgskole 53, 1-19, Report No. 190.

MASSON, M. F. 1985. Mapping, Combining Abilities, Heritabilities and Heterosis with $4 \mathrm{x} \times 2 \mathrm{x}$ Crosses in Potato. Ph.D. Thesis, University of Wisconsin.

SALAMAN, R. N. 1910. The inheritance of colour and other characters in the potato. J. Genet., 1, 7-46.

Salaman, R. N. 1926. Potato Varieties. Cambridge University Press, London.

STAM, P. 1993. Construction of integrated genetic linkage maps by means of a new computer package, JOINMAP. Plant J., 3, 739-744.
SWAMINATHAN, M. S. AND HOWARD, H. W. 1953. The cytology and genetics of the potato (Solanum tuberosum) and related species. Biblio. Genet., 16, 1-192.

UPOV 1986. Guidelines for the conduct of tests for distinctness, homogeneity and stability. Potato. Union Internationale pour la Protection des Obtentions Végétales document TG/23/5.

VAN ECK, H. J., JACOBS, J. M. E., VAN DIJK, J., STIEKEMA, W. J. AND JACOBSEN, E. 1993. Identification and mapping of three flower colour loci of potato ( $S$. tuberosum) by RFLP analysis. Theor. Appl. Genet., 86, 295-300.

VAN ECK, H. J., JACOBS, J. M. E., STAM, P., TON, J., STIEKEMA, W. J. AND JACOBSEN, E. 1994. Multiple alleles for tuber shape in diploid potato detected by qualitative and quantitative genetic analysis using RFLPs. Genetics, 137, 303-309.

VAN OOIJEN, J. W. 1994. DRAWMAP: A computer program for drawing genetic linkage maps. J. Hered., 85, 66.

VON RATHLEF, H. AND SIEBENEICK, H. 1934. Ueber einige Kreuzungen peruanischer Sorten von $S$. andigenum Juz. et Buk. mit Richters Jubel und die Genetik von Schalenfarbe, Knollenfarbe, Fleischfarbe, Blütenfarbe, und Knollenform bei der Kartoffel. Genetica, 16, 153-176.

WU, K. K., BURNQUIST, W., SORRELS, M. E., TEW, T. L., MOORE, P. H. AND TANKSLEY, S. D. 1991. The detection and estimation of linkage in polyploids using single dose restriction fragments. Theor. Appl. Genet., 83, 294-300. 